\title{
Gastrointestinal transit time and heart rate variability in patients with mild acquired brain injury
}

\author{
Johannes Enevoldsen ${ }^{\text {Corresp., }}{ }^{1}$, Simon T Vistisen ${ }^{2}$, Klaus Krogh ${ }^{3}$, Jørgen F Nielsen ${ }^{4}$, Karoline Knudsen ${ }^{5}$, Per \\ Borghammer $^{5}$, Henning Andersen ${ }^{1}$ \\ 1 Department of Neurology, Aarhus University Hospital, Aarhus, Denmark \\ 2 Research Centre for Emergency Medicine, Aarhus University, Aarhus, Denmark \\ 3 Neurogastroenterology Unit, Department of Hepatology and Gastroenterology, Aarhus Univeristy Hospital, Aarhus, Denmark \\ 4 Hammel Neurocenter and University Clinic, Aarhus University, Aarhus, Denmark \\ 5 Department of Nuclear Medicine \& PET-Centre, Aarhus University Hospital, Aarhus, Denmark \\ Corresponding Author: Johannes Enevoldsen \\ Email address: johene@rm.dk
}

Background. Constipation is suspected to occur frequently after acquired brain injury (ABI). In patients with $A B I$, heart rate variability (HRV) is reduced suggesting autonomic dysfunction. Autonomic dysfunction may be associated with prolonged gastrointestinal transit time (GITT). The primary aim of this study was to investigate if GITT is prolonged in patients with ABI. Secondarily, HRV and its correlation with GITT was investigated.

Methods. We included 25 patients with ABI (18 men, median age: 61.3 years (range: 30.7-74.5)). GITT was assessed using radio-opaque markers and HRV was calculated from 24-hour electrocardiograms. Medical records were reviewed for important covariates, including primary diagnosis, time since injury, functional independence measure, and use of medication. The GITT assessed in patients was compared to a control group of 25 healthy subjects (18 men, median age: 61.5 years (range: $34.0-70.9$ )).

Results. In ABI patients, the mean GITT was significantly longer than in healthy controls ( 2.68 days, $95 \%$ $\mathrm{Cl}[2.16 ; 3.19]$ versus $(1.92$ days, $95 \% \mathrm{Cl}[1.62 ; 2.22], \mathrm{p}=0.011)$. No correlation was found between HRV and GITT.

Conclusion. Patients with mild to moderate $A B I$ have prolonged GITT unrelated to the HRV. 


\section{Gastrointestinal transit time and heart rate}

2 variability in patients with mild acquired brain

3 injury

4 Johannes Enevoldsen ${ }^{1}$, Simon T Vistisen ${ }^{2}$, Klaus Krogh ${ }^{3}$, Jørgen F Nielsen ${ }^{4}$, Karoline Knudsen ${ }^{5}$,

5 Per Borghammer ${ }^{5}$, Henning Andersen ${ }^{1}$

$6 \quad{ }^{1}$ Department of Neurology, Aarhus University Hospital, Denmark

$7 \quad{ }^{2}$ Research Centre for Emergency Medicine, Institute of Clinical Medicine, Aarhus University,

8 Denmark

$9 \quad{ }^{3}$ Neurogastroenterology Unit, Department of Hepatology and Gastroenterology, Aarhus

10 University Hospital, Denmark

$11{ }^{4}$ Hammel Neurocenter and University Clinic, Denmark

$12{ }^{5}$ Department of Nuclear Medicine \& PET-Centre, Aarhus University Hospital, Denmark

13 Corresponding author

14 Johannes Enevoldsen ${ }^{1}$,

15 Nørrebrogade 44, 8000 Aarhus C, Denmark

16 Phone: +4528405305, Email: johannes.ne@gmail.com 


\section{Abstract}

18 Background. Constipation is suspected to occur frequently after acquired brain injury (ABI). In

19 patients with $\mathrm{ABI}$, heart rate variability (HRV) is reduced suggesting autonomic dysfunction.

20 Autonomic dysfunction may be associated with prolonged gastrointestinal transit time (GITT).

21 The primary aim of this study was to investigate if GITT is prolonged in patients with ABI.

22 Secondarily, HRV and its correlation with GITT was investigated.

23 Methods. We included 25 patients with ABI (18 men, median age: 61.3 years (range: $30.7-$

24 74.5)). GITT was assessed using radio-opaque markers and HRV was calculated from 24-hour

25 electrocardiograms. Medical records were reviewed for important covariates, including primary

26 diagnosis, time since injury, functional independence measure, and use of medication. The GITT

27 assessed in patients was compared to a control group of 25 healthy subjects (18 men, median

28 age: 61.5 years (range: $34.0-70.9)$ ).

29 Results. In ABI patients, the mean GITT was significantly longer than in healthy controls $(2.68$

30 days, 95\% CI [2.16; 3.19] versus (1.92 days, 95\% CI [1.62; 2.22], $\mathrm{p}=0.011)$. No correlation was

31 found between HRV and GITT.

32 Conclusion. Patients with mild to moderate ABI have prolonged GITT unrelated to the HRV. 


\section{Introduction}

35 Acquired brain injury (ABI) is a frequent cause of mortality and disability. In 2009, the

36 incidence of hospitalizations due to ABI was approximately 400 per 100,000 in Denmark

37 (National Board of Health, 2011), and in 2012, the mean European incidence of hospitalizations

38 due to traumatic brain injury (TBI) was 287.2 per 100,000 (Majdan et al., 2016).

39 Constipation and prolonged gastrointestinal transit time (GITT) are very common complications

40 to acute and chronic spinal cord lesions (Han, Kim \& Kwon, 1998; Krogh, Mosdal \& Laurberg,

41 2000), but little is known about bowel function after ABI.

42 Two studies including Korean patients with ABI or stroke found that GITT was associated to

43 subjective symptoms of constipation (Lim et al., 2012; Yi et al., 2011). However, none of the

44 studies included a healthy control group. Other data indicate that gastric emptying is delayed in

45 patients with TBI (Kao et al., 1998). In animal studies, contractile activity was reduced and

46 transit time of the small intestine was prolonged after TBI (Olsen et al., 2013; Wang, Liu \&

47 Yang, 2011). A study, comparing patients with stroke to patients with orthopedic injuries and

48 similar immobility, showed that patients with stroke had a higher risk of de novo constipation

49 (assessed by the Rome II questionnaire) (Bracci et al., 2007). This suggests that ABI causes

50 bowel dysfunction. It is likely that impaired gastrointestinal motility may be caused, at least in

51 part, by injuries to the central autonomic nervous system. Other causes of gastrointestinal

52 dysmotility after ABI may include immobility and side effects of medication.

Autonomic function can be assessed, non-invasively, by measuring heart rate variability (HRV).

54 High frequency variability in the heart rate is mediated primarily by changes in the

55 parasympathetic (vagal) tone on the heart (AHA and ESC, 1996; Metelka, 2014). The

56 parasympathetic nervous system modulates bowel motility also (Furness, 2012), and as recent 
57 studies reported that HRV is lowered in patients with ABI (Keren et al., 2005; King et al., 1997;

58 Vistisen et al., 2014), it is relevant to investigate the correlation between HRV and GITT in these

59 patients.

60 To enable identification of residual gastrointestinal symptoms or impaired motility, despite

61 presumed optimal laxative treatment, the aim of this study was to compare GITT in patients with

$62 \mathrm{ABI}$ and healthy control subjects. Furthermore, we aimed to investigate whether there is an

63 association between GITT and HRV in patients with ABI. 


\section{Method}

\section{Subjects}

66 This study was approved by the regional research ethics committee (application no: 49007) and

67 by the Data Protection Agency (jr.no: 2007-58-0010). The study was registered at

68 ClinicalTrials.gov (identifier: NCT02428790).

69 Twenty-five patients undergoing rehabilitation for ABI were included from Hammel

70 Neurocenter and University Clinic between June and November 2015.

71 Inclusion criteria were: Age 18 to 79 years, ABI within one year before inclusion (i.e. stroke

72 including subarachnoid haemorrhage, anoxic brain injury or moderate to severe TBI (Glasgow

73 Coma Scale (GCS) score 3-12 at admission to the intensive care unit)), ability to swallow the

74 capsules used to assess GITT and sufficient cognitive performance to give informed consent.

75 Exclusion criteria were: Major abdominal disorders, severe acute comorbidity, cancer,

76 pregnancy, autonomic neuropathy, or other known neurological disorder.

77 Informed consent was obtained.

78 Control subjects were recruited from two historic datasets from our unit including 38 subjects.

79 One set consisted of healthy hospital and university employees (a subset of these have previously

80 been published (Krogh et al., 2000)), and the other consisted of an elderly control group,

81 matched to a study population of patients with Parkinson's disease (Knudsen et al., 2017). No

82 control subject used prescription laxatives or had gastrointestinal diseases. Control subjects were

83 selected blindly from the two datasets to match the age and sex of the patients with ABI on a

84 group level, using the following method: From the total set of control subjects, subjects older 
85 than 79 years were excluded. The set was split in males and females and ordered by age. With a

86 ratio of 2.6 males per female (as in the patient cohort), the oldest control subjects were selected

87 until the median age matched the patient cohort.

88 Gastrointestinal transit time

89 Total GITT was assessed with radio-opaque markers as described by (Abrahamsson, Antov \&

90 Bosaeus, 1988). The measurement is a 7 day procedure, where the patients, on each of day 1-6,

91 ingest one capsule containing ten radio-opaque markers (Colon Transit, REF:CTT6V10). An X-

92 ray examination of the abdomen is performed on day 7. Ingestion of capsules and the X-ray

93 examination was done at noon, except for one patient, who, for convenience, had both done at 8

94 am.

95 GITT was estimated using the published equation: GITT $=\left(\mathrm{M}+0.5^{*} \mathrm{D}\right) / \mathrm{D}$, in which GITT is the

96 gastrointestinal transit time in days, $\mathrm{M}$ is the number of markers counted on the $\mathrm{X}$-ray and $\mathrm{D}$ is

97 the daily dose of markers $(\mathrm{D}=10)$.

98 In other publications, GITT is often denoted as colonic transit time (CTT). However, the method

99 assesses the transit time of the entire gastrointestinal system, with colonic transit time accounting

100 for the majority (Degen, Phillips \& Izzo, 1996).

101 Neurogenic bowel dysfunction score

102 After the end of the inclusion period, one of the authors (JE) contacted the participants, and

103 performed a questionnaire regarding their bowel function, using the Neurogenic Bowel

104 Dysfunction (NBD) score (Krogh et al., 2006). Patients who were still admitted were contacted 
105 in person, while discharged patients were contacted by phone. In both cases, the interviewer read

106 the questions aloud and registered the patient's answers.

\section{Heart rate variability}

108 Twenty-four hour electrocardiograms (ECGs) were measured using a wearable 1-lead device

109 (eMotion Faros 90, Mega Electronics Ltd, Kuopio, Finland), using a sampling rate of $250 \mathrm{~Hz}$.

110 The recordings where performed during the week preceding the X-ray examination.

111 Heart rate variability measures were calculated from the 24-hour ECG using Kubios HRV

112 (University of Eastern Finland, Kuopio, Finland) (Tarvainen et al., 2009). The methods comply

113 with the HRV guideline provided by the Task Force of The European Society of Cardiology and

114 The North American Society of Pacing and Electrophysiology (AHA and ESC, 1996). For each

115 patient, a 24-hour analysis and four 5 min analyses were performed.

116 The 24-hour analysis included all normal-to-normal intervals (NN). Initial identification of

117 normal beats was performed, using the automatic R wave detection in Kubios HRV. Then

118 manual correction of erroneous or missing R wave markings was performed and ectopic beats

119 were removed. Artefact correction was applied to remove the resulting non-normal intervals. To

120 optimally distinguish between normal and non-normal intervals, individual correction strengths

121 were applied to each ECG recording.

122 The 5 min analyses were performed approximately at 6 am, $1 \mathrm{pm}, 6 \mathrm{pm}$ and $2 \mathrm{am}$. These time

123 points were chosen because no rehabilitation training was scheduled at these time points, and the

124 patients would usually be physically inactive. Patients were not observed during these 5 min

125 intervals. For each analysis, a 5 min section of the ECG, with little or no non-normal beats and

126 no upwards or downwards trend in RR duration, was selected. Any non-normal beats were 
127 removed manually, and the resulting non-normal intervals were removed using the lowest ('very

128 low') artefact correction setting.

129 Non-parametric calculations (i.e. fast Fourier transforms (FFT)) were used for frequency domain

130 analyses. Standard ranges for low frequency (LF) and high frequency (HF) parts of the spectrum

131 were used (i.e. LF, 0.04-0.15 Hz; HF, 0.15-0.4 Hz).

132 The main HRV measures were chosen to be the root mean square of successive NN differences

133 (RMSSD) and HF power. These measures are believed to reflect mainly the parasympathetic

134 (vagal) component of the regulation of the heart rate. Other HRV variables are reported for 135 comparison with other studies.

\section{Covariates}

137 Covariates, including time of injury, type of injury, medication and Functional Independence

138 Measure (FIM), were acquired from the medical records.

139 Medication use, either scheduled or pro re nata, was registered based on patients' medical

140 records on the day of the X-ray examination. Use of oral laxatives, opioid analgesics, statins,

141 selective serotonin reuptake inhibitors (SSRI), and baclofen were registered.

142 Functional ability of the patients was assessed using FIM. FIM is used for routine scoring at our

143 rehabilitation hospital, and reflects the level of independence in everyday tasks (Uniform Data

144 System for Medical Rehabilitation, 2012). Scoring is performed approximately once per month

145 by the team of treating doctors, nurses, physiotherapists and occupational therapists. In this

146 study, we used the FIM scores performed in closest temporal proximity to the X-ray

147 examination. 


\section{Statistical analyses}

149 Results are presented as mean [95\% CI], unless otherwise indicated. The difference in GITT and

150 age between patients and healthy control subjects was tested using a Welch t-test due to unequal

151 variance. Differences in SD were tested using F-tests, and proportions using Pearson's $\chi^{2}$ test.

152 Linear regression modeling GITT as a function of group (patient vs control), age, and sex was

153 performed.

154 Correlations including time since injury (at the day of the X-ray examination) and FIM were

155 calculated using Spearman's rank correlation ( $\rho)$, while correlations between GITT, age and

156 HRV measures were calculated using Pearson product-moment correlation coefficient (r).

157 Comparison of GITT in users with non-users of medication (laxatives, opioids, statins and

158 SSRIs) was done using confidence intervals based on the t-distribution. Between users and non-

159 users of laxatives, differences in FIM, time since injury and age were tested using the Wilcoxon-

160 Mann-Whitney test.

161 Heart rate variability measures for the four 5 min HRV analyses were summarised for each

162 patient (the four analyses are one for each time point). For HRV measures (not including heart

163 rate (HR)) logarithmic transformation was performed prior to calculation of summary statistics,

164 correlations and regressions. Therefore, the reported means are geometric for all HRV measures

165 and arithmetic for HR.

166 Our power calculation was based on the standard deviation (SD) of GITT among healthy

167 subjects. We assumed that SD of GITT among the patients with ABI would be identical. The

168 target difference in GITT was 1 day, and with a significance level $(\alpha)$ of $0.05,11$ patients and 11

169 control subject would be necessary to reach a statistical power of 0.90 . 
170 To ensure sufficient statistical power despite dropouts or higher SD among patients, and to make

171 statistical corrections for age and sex possible, we aimed to include 30 patients with ABI.

172 Statistical analyses and calculations apart from HRV analysis, was performed using R 3.4.3 (R

173 Core Team, 2017) with additional packages (Bengtsson, 2015; Fischer \& Pau, 2015; Grolemund

174 \& Wickham, 2011; Hlavac, 2015; Robinson, 2015; Wickham, 2007, 2009; Wickham \& Francois, 175 2016). 


\section{Results}

177 During the inclusion period, 103 patients were screened for inclusion. Of these, 45 were eligible

178 for inclusion. Twenty-six patients were included in the study, of which one was later excluded

179 due to an erroneous ABI diagnosis (figure 1).

180 The mean GITT of the 25 patients with ABI was 2.68 [2.17; 3.19] days. The 25 healthy control

181 subjects had a lower GITT of $1.92[1.62 ; 2.22]$ days $(\mathrm{p}=0.011)$. Also, the patients had a higher

182 SD of GITT than the control subjects $(1.24$ and 0.73 days, $\mathrm{p}=0.011)($ table 1$)$.

183 Linear regression of GITT by group (patient vs control), age and sex showed that ABI and higher

184 age were associated with longer GITT, while sex was not a predictor (table 2 and figure 2).

185 The median time from X-ray examination to FIM scoring was -1 day, IQR $[-13 ; 6]$. Patients

186 with ABI had a median FIM score of 111, IQR [97; 116]. FIM score did not correlate with age ( $\rho$

$187=-0.21, \mathrm{p}=0.31)$ or $\operatorname{GITT}(\rho=-0.32, \mathrm{p}=0.12)$

188 The median time since injury was 71 days, IQR $[39 ; 131]$. Time since injury did not correlate

189 with GITT $(\rho=-0.06, p=0.77)$ or $\operatorname{FIM}(\rho=0.02, p=0.91)$

190 Twelve (48\%) patients with ABI received oral laxatives. GITT for these patients were not

191 significantly longer than GITT for patients who did not use oral laxatives $(+0.33[-0.71 ; 1.37]$

192 days). There was no significant difference in age $(-1.1$ years, $p=0.77)$ or time since injury

193 ( -29.6 days, $p=0.22$ ) between the two groups (laxative users - non-users). Laxative users did

194 have a significantly lower FIM score (difference: 19.7, p=0.02), which remained significant

195 after subtraction of the bowel function subscore from the total FIM score (difference: 18.6, $\mathrm{p}=$

196 0.02). Of the 13 patients with $\mathrm{ABI}$ who did not have a prescription for oral laxatives, $10(77 \%)$

197 received an oral laxative at some point between their injury and the X-ray examination. 
198 Opioid analgesics, statins and SSRIs were used by $4(16 \%), 11(44 \%)$ and $8(32 \%)$ of the

199 patients respectively. None of the patients used baclofen. Neither opioid, statin nor SSRI users

200 had a longer GITT than patients who did not use these medications (opioid users, -0.42 [ -1.84 ;

$2010.99]$ days; statin users, 0.60 [ $-0.42 ; 1.63]$ days; SSRI users, $0.36[-0.75 ; 1.47]$ days).

\section{Neurogenic bowel dysfunction score}

203 The neurogenic bowel dysfunction score (NBD) was completed by 23 patients with ABI. The

204 median time from injury to completion of the questionnaire was 163 days, range [38; 395] and

205 the median time from the X-ray examination to completion of the questionnaire was 82 days, 206 range $[8 ; 176]$.

207 One patient with ABI had a 'moderate' bowel dysfunction (NBD score $=10)$ and reported that 208 her gastrointestinal function had 'some' impact on her quality of life. The remaining 22 patients 209 had either no or 'very minor' bowel dysfunction (mean NBD score $=0.5$, range $[0 ; 5]$ ) and 210 reported that this had 'no' or 'little' impact on quality of life.

\section{Heart rate variability}

212 The geometric mean HF power of the 5 min analyses of ECGs was 90.7 [50.5; 162.6] $\mathrm{ms}^{2}$. The

213 geometric mean RMSSD was 18.1 [13.4; 24.6] ms. No significant correlation was found between

214 GITT and the logarithm of HF power $(r=-0.117[-0.490 ; 0.292])$ or GITT and the logarithm of

215 RMSSD $(\mathrm{r}=-0.119[-0.491 ; 0.290])$. One patient had several periods with arrhythmia, making

216 only 5 min analyses possible. Further results from the HRV analyses are shown in table 3 and 4. 


\section{Discussion}

218 This study aimed to characterise GITT in patients with ABI and compare findings to healthy

219 control subjects. Previous studies in patients with ABI, although using similar methodology, did

220 not perform a direct comparison to healthy control subjects (Lim et al., 2012).

221 Patients with ABI had significantly longer GITT than healthy control subjects, but with

222 substantial overlap. While the mean GITT of 2.68 days is significantly longer than normal, it is

223 only modestly increase compared with the mean GITT of $>4$ days found in patients with acute

224 spinal cord lesions (Krogh et al., 2000). The NBD score revealed limited clinical bowel

225 dysfunction in the patient group. This is surprising compared to a study of de novo constipation

226 on hemiplegic stroke patients, which found constipation in 30\% of patients (Bracci et al., 2007).

227 Their time from injury to interview was longer (254 days, IQR [138; 565] against 163 days, IQR

$228[109 ; 213]$ in our study). However, only $24.4 \%$ of patients used laxatives, against $48 \%$ in our

229 study, and the questionnaire used to assess bowel function (Rome II) was different. Advanced

230 age was related to an increased GITT, which is a common, though not entirely consistent, finding

231 in other studies (Graff, Brinch \& Madsen, 2001).

232 There are large global differences in GITT, with western populations having a markedly longer

233 mean transit time than Asian (Jung, Kim \& Moon, 2003). Since alternate and shorter protocols

234 are often used for measuring GITT in populations with faster transit times, direct comparisons

235 among studies are difficult (Ghoshal, Sengar \& Srivastava, 2012). The healthy control subjects

236 presented in our study had a mean GITT similar to that reported in a Swedish study

237 (Abrahamsson et al., 1988). On the other hand, Lim et al., 2012 showed that Korean patients

238 with ABI, who were clinically constipated, had a GITT similar to the healthy control subjects in

239 our study. The mean GITT was 1.94 days in Korean patients with ABI and 1.92 days in our 
240 healthy control subjects (Lim et al., 2012). Thus, the inclusion of local control subjects seems

241 mandatory in GITT studies.

242 We found no correlation between HRV and GITT which may be due to low statistical power.

243 The use of laxatives may have hidden any correlation by narrowing the variability in GITT. It is

244 also possible that the correlation does not exist. The HRV variables, HF and RMSSD,

245 predominantly reflect parasympatic (vagal) tone on the sinoatrial node. The enteric nervous

246 system, which controls gastrointestinal motility, responds not only to parasympathetic input but

247 also to local and regional stimulation such as mechanical stretch of the intestinal wall and

248 endocrine hormones. Such regional and local stimulation may be more pronounced determinants

249 of gastrointestinal motility than parasympathetic tone, thereby masking any correlations with

$250 \mathrm{HRV}$. It would be important to investigate the correlation between GITT and HRV in more

251 severely injured patients with ABI.

252 In a previous study from our rehabilitation hospital we compared HRV in 49 patients with ABI

253 with 49 healthy control subjects (Vistisen et al., 2014). The patients with ABI were included

254 from the semi-intensive care rehabilitation unit. They were all bedridden and had a markedly

255 lower FIM score. The mean HRV of the patients in the present study lie between the patients and

256 the controls from the previous study, which suggests a less severe autonomic dysfunction in the

257 present patients as compared to more severely injured patients with ABI (HF power: Healthy

258 controls, $290[211 ; 399] \mathrm{ms}^{2}$; patients in the current study, $90.7[50.5 ; 162.6] \mathrm{ms}^{2}$; patients in

259 semi-intensive care rehabilitation, $\left.8.9[5.4 ; 14.8] \mathrm{ms}^{2}\right)$.

260 Some limitations need to be addressed. 
261 First, for ethical reasons, we accepted laxative use during the GITT measurement, among the 262 patients with ABI. This may have resulted in an underestimation of the difference in GITT

263 between patients and healthy control subjects.

264 Second, we did not include patients with dysphagia or mental impairment. Thereby only patients

265 with milder ABI or late in their rehabilitation have been included. Adding to this, patients with

266 more severe illness may be less likely to participate, due to fatigue. Indeed, our rehabilitation

267 centre treats patients who are generally more disabled than the cohort in this study (Stubbs et al.,

268 2014). The results are therefore not necessarily representative of patients in the acute phase after

269 ABI and patients with severe disability.

270 Third, though we did exclude patients with autonomic neuropathy or other neurological diseases

271 apart from ABI, patients were not examined for unrecognised neuropathy. As diabetic patients

272 are at risk of both stroke and autonomic neuropathy, this may have contributed to the prolonged

273 GITT and low HRV observed in these patients. The prevalence of diabetes in our study

274 population is unknown.

275 Other limitations include the heterogeneity of the patients included, the late application of the

276 bowel dysfunction questionnaire and some characteristics about the HRV measurement

277 discussed below. We have not reported the severity of patients' injuries at presentation, as

278 different severity scores are used for each group of injuries. Also, severity scores were not

279 available for all patients. We believe that the FIM score is a good indicator of the patients'

280 condition at the time of GITT measurement.

281 Heart rate variability can be confounded by endogenous factors. Most importantly, respiratory

282 HRV components (i.e. HF and RMSSD) are lowered with increasing respiratory rate and heart 
283 rate (Weippert et al., 2015). To minimise the effect of physical activity on HRV, the 5 min

284 analyses were performed on ECG recordings of periods, where the patients were scheduled to be 285 resting.

286 This study does not cover whether the bowel dysfunction is a direct effect of the ABI or

287 secondary to the changes in daily living following ABI (e.g. immobility and changes in diet). It

288 may well be a combination and the clinical issue is important to address regardless of the 289 causation.

290 We consider that prolonged GITT, despite common use of laxatives, support the clinical practice 291 of laxative prescription to patients with mild impairment after ABI. However, the observational 292 nature of this study does not prove the usefulness of laxatives in this patient group. We hope this 293 new evidence on bowel function patients with ABI will reinforce the importance of managing 294 gastrointestinal impairment in these patients.

\section{Conclusion}

296 Gastrointestinal transit time (GITT) was significantly prolonged in patients with mild

297 impairment after ABI compared with healthy controls. The difference may have been

298 underestimated due to laxative use in half of the patients. No correlation between GITT and

299 HRV was found. Further studies are needed to assess bowel function in the acute phase after 300 ABI.

\section{Acknowledgements}

302 Thanks to Department of Radiology, Regional Hospital Silkeborg, for support and education and 303 to the ward staff at Hammel Neurocenter and University Clinic for invaluable help with the day304 to-day operation of the study. 
305 References

306 Abrahamsson H, Antov S, Bosaeus I. 1988. Gastrointestinal and colonic segmental transit time

307 evaluated by a single abdominal x-ray in healthy subjects and constipated patients. Scandinavian

308 Journal of Gastroenterology. 23(suppl 152):72-80 DOI:10.3109/00365528809095938

309 AHA and ESC. 1996. Heart rate variability. Standards of measurement, physiological

310 interpretation, and clinical use. Task Force of the European Society of Cardiology and the North

311 American Society of Pacing and Electrophysiology. European Heart Journal. 17(3):354-381

312 Retrieved from http://www.ncbi.nlm.nih.gov/pubmed/8737210

313 Bengtsson H. 2015. R.matlab: Read and Write MAT Files and Call MATLAB from Within R

314 Retrieved from http://cran.r-project.org/package=R.matlab

315 Bracci F, Badiali D, Pezzotti P, Scivoletto G, Fuoco U, Di Lucente L, Petrelli a, Corazziari E.

316 2007. Chronic constipation in hemiplegic patients. World Journal of Gastroenterology: WJG.

317 13(29):3967-3972

318 Degen LP, Phillips SF, Izzo D. 1996. Variability of gastrointestinal transit in healthy women and

319 men. Gut. 39(2):299-305

320 Fischer B, Pau G. 2015. rhdf5: HDF5 interface to R

321 Furness JB. 2012. The enteric nervous system and neurogastroenterology. Nature Reviews

322 Gastroenterology \& Hepatology. 9(5):286-294 DOI:10.1038/nrgastro.2012.32

323 Ghoshal UC, Sengar V, Srivastava D. 2012. Colonic transit study technique and interpretation:

324 Can these be uniform globally in different populations with non-uniform colon transit time?

325 Journal of Neurogastroenterology and Motility. 18(2):227-228 DOI:10.5056/jnm.2012.18.2.227

326 Graff J, Brinch K, Madsen JL. 2001. Gastrointestinal mean transit times in young and middle-

327 aged healthy subjects. Clinical Physiology. 21(2):253-259 DOI:10.1046/j.1365-

$328 \quad 2281.2001 .00308 . x$

329 Grolemund G, Wickham H. 2011. Dates and Times Made Easy with \{lubridate\}. Journal of

330 Statistical Software. 40(3):1-25 Retrieved from http://www.jstatsoft.org/v40/i03/

331 Han TR, Kim JH, Kwon BS. 1998. Chronic gastrointestinal problems and bowel dysfunction in

332 patients with spinal cord injury. Spinal Cord. 36(7):485-490 DOI:10.1038/sj.sc.3100616

333 Hlavac M. 2015. stargazer: Well-Formatted Regression and Summary Statistics Tables.

334 Cambridge, USA Retrieved from http://cran.r-project.org/package=stargazer

335 Jung H, Kim D, Moon I. 2003. Effects of gender and menstrual cycle on colonic transit time in

336 healthy subjects. The Korean Journal of Internal Medicine. 18:181-186

337 Kao C-H, ChangLai S-P, Chieng P-U, Yen T-C. 1998. Gastric emptying in head-injured patients.

338 The American Journal of Gastroenterology. 93(7):1108-1112 DOI:10.1111/j.1572-

339 0241.1998.00338.x

340 Keren O, Yupatov S, Radai MM, Elad-Yarum R, Faraggi D, Abboud S, Ring H, Groswasser Z.

341 2005. Heart rate variability (HRV) of patients with traumatic brain injury (TBI) during the post-

342 insult sub-acute period. Brain Injury. 19(8):605-611 DOI:10.1080/02699050400024946 
343 King ML, Lichtman SW, Seliger G, Ehert F a, Steinberg JS. 1997. Heart-rate variability in

344 chronic traumatic brain injury. Brain Injury. 11(6):445-453

345 Knudsen K, Fedorova TD, Bekker AC, Iversen P, Østergaard K, Krogh K, Borghammer P. 2017.

346 Objective Colonic Dysfunction is Far more Prevalent than Subjective Constipation in

347 Parkinson's Disease: A Colon Transit and Volume Study. Journal of Parkinson's Disease. 1-9

348 DOI:10.3233/JPD-161050

349 Krogh K, Christensen P, Sabroe S, Laurberg S. 2006. Neurogenic bowel dysfunction score.

350 Spinal Cord. 44(10):625-631 DOI:10.1038/sj.sc.3101887

351 Krogh K, Mosdal C, Laurberg S. 2000. Gastrointestinal and segmental colonic transit times in

352 patients with acute and chronic spinal cord lesions. Spinal Cord. 38(10):615-621 Retrieved from

353 http://www.ncbi.nlm.nih.gov/pubmed/11093323

354 Lim YH, Kim DH, Lee MY, Joo MC. 2012. Bowel Dysfunction and Colon Transit Time in

355 Brain-Injured Patients. Annals of Rehabilitation Medicine. 36(3):371-378

356 DOI:10.5535/arm.2012.36.3.371

357 Majdan M, Plancikova D, Brazinova A, Rusnak M, Nieboer D, Feigin V, Maas A. 2016.

358 Epidemiology of traumatic brain injuries in Europe : a cross-sectional analysis. The Lancet

359 Public Health. 1(2):e76-e83 DOI:10.1016/S2468-2667(16)30017-2

360 Metelka R. 2014. Heart rate variability - current diagnosis of the cardiac autonomic neuropathy.

361 A review. Biomedical Papers of the Medical Faculty of the University Palacky, Olomouc,

362 Czechoslovakia. 158 DOI:10.5507/bp.2014.025

363 National Board of Health. 2011. Braininjury Rehabilitation - a health technology assessment.

364 summary. National Board of Health, Danish Centre of Health Technology Assessment. 1(1)

365 Olsen AB, Hetz RA, Xue H, Aroom KR, Bhattarai D, Johnson E, Bedi S, Cox CS, Uray K. 2013.

366 Effects of traumatic brain injury on intestinal contractility. Neurogastroenterology \& Motility.

367 25(7):593-e463 DOI:10.1111/nmo.12121

368 R Core Team. 2017. R: A Language and Environment for Statistical Computing. Vienna, Austria

369 Retrieved from https://www.r-project.org/

370 Robinson D. 2015. broom: Convert Statistical Analysis Objects into Tidy Data Frames Retrieved

371 from http://cran.r-project.org/package=broom

372 Stubbs PW, Pallesen H, Pedersen AR, Nielsen JF. 2014. Using EFA and FIM rating scales could 373 provide a more complete assessment of patients with acquired brain injury. Disability and

374 Rehabilitation. 36(26):2278-2281 DOI:10.3109/09638288.2014.904935

375 Tarvainen MP, Niskanen J-P, Lipponen J a, Ranta-aho PO, Karjalainen P a. 2009. Kubios HRV 376 A Software for Advanced Heart Rate Variability Analysis. 4th European Conference of the

377 International Federation for Medical and Biological Engineering. 1(3):1022-1025

378 DOI:10.1007/978-3-540-89208-3_243

379 Uniform Data System for Medical Rehabilitation. 2012. The FIM ${ }^{\circledR}$ Instrument: Its Background, 380 Structure, and Usefulness. Buffalo (NY) Retrieved from

381 www.udsmr.org/Documents/The_FIM_Instrument_Background_Structure_and_Usefulness.pdf 
382 Vistisen ST, Hansen TK, Jensen J, Nielsen JF, Fleischer J. 2014. Heart rate variability in

383 neurorehabilitation patients with severe acquired brain injury. Brain Injury. 28(2):196-202

384 DOI:10.3109/02699052.2013.860477

385 Wang Y-B, Liu J, Yang Z-X. 2011. Effects of intestinal mucosal blood flow and motility on

386 intestinal mucosa. World Journal of Gastroenterology : WJG. 17(5):657-661

387 DOI:10.3748/wjg.v17.i5.657

388 Weippert M, Behrens K, Rieger A, Kumar M, Behrens M. 2015. Effects of breathing patterns

389 and light exercise on linear and nonlinear heart rate variability. Applied Physiology, Nutrition,

390 and Metabolism. 40(8):762-768 DOI:10.1139/apnm-2014-0493

391 Wickham H. 2007. Reshaping Data with the \{reshape\} Package. Journal of Statistical Software.

392 21(12):1-20 Retrieved from http://www.jstatsoft.org/v21/i12/

393 Wickham H. 2009. ggplot2: elegant graphics for data analysis. Springer New York Retrieved

394 from http://had.co.nz/ggplot2/book

395 Wickham H, Francois R. 2016. dplyr: A Grammar of Data Manipulation Retrieved from

396 https://cran.r-project.org/package=dplyr

397 Yi JH, Chun MH, Kim BR, Han EY, Park JY. 2011. Bowel Function in Acute Stroke Patients.

398 Annals of Rehabilitation Medicine. 35(3):337-343 DOI:10.5535/arm.2011.35.3.337 
Figure 1

Flow chart of patients with acquired brain injury ( $A B I)$ included in the present study.

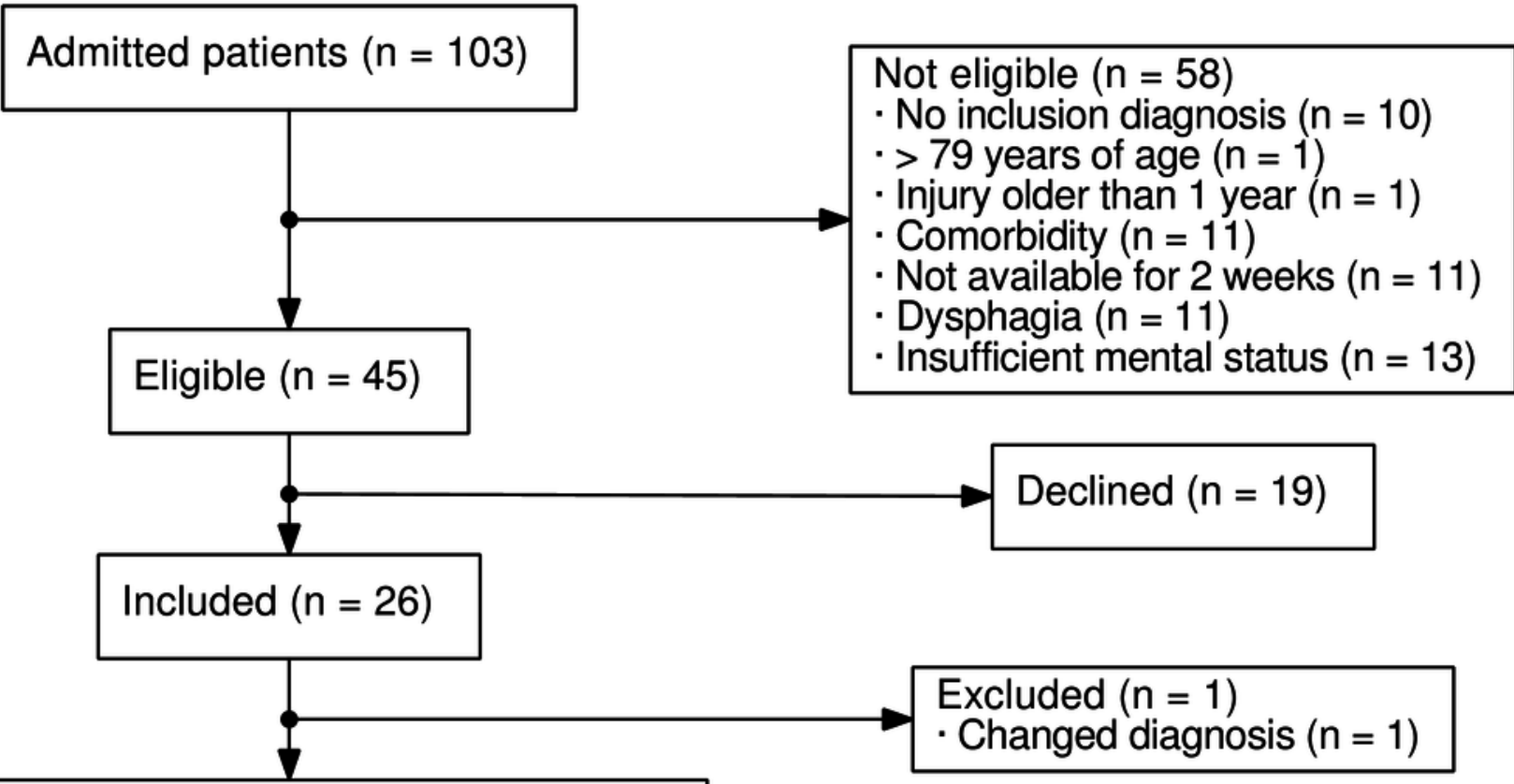

Completed clinical measurement $(n=25)$

Completed bowel dysfunction questionnaire $(n=23)$ 
Figure 2

Gastrointestinal transit time (GITT) and age of patients with acquired brain injury and healthy control subjects.

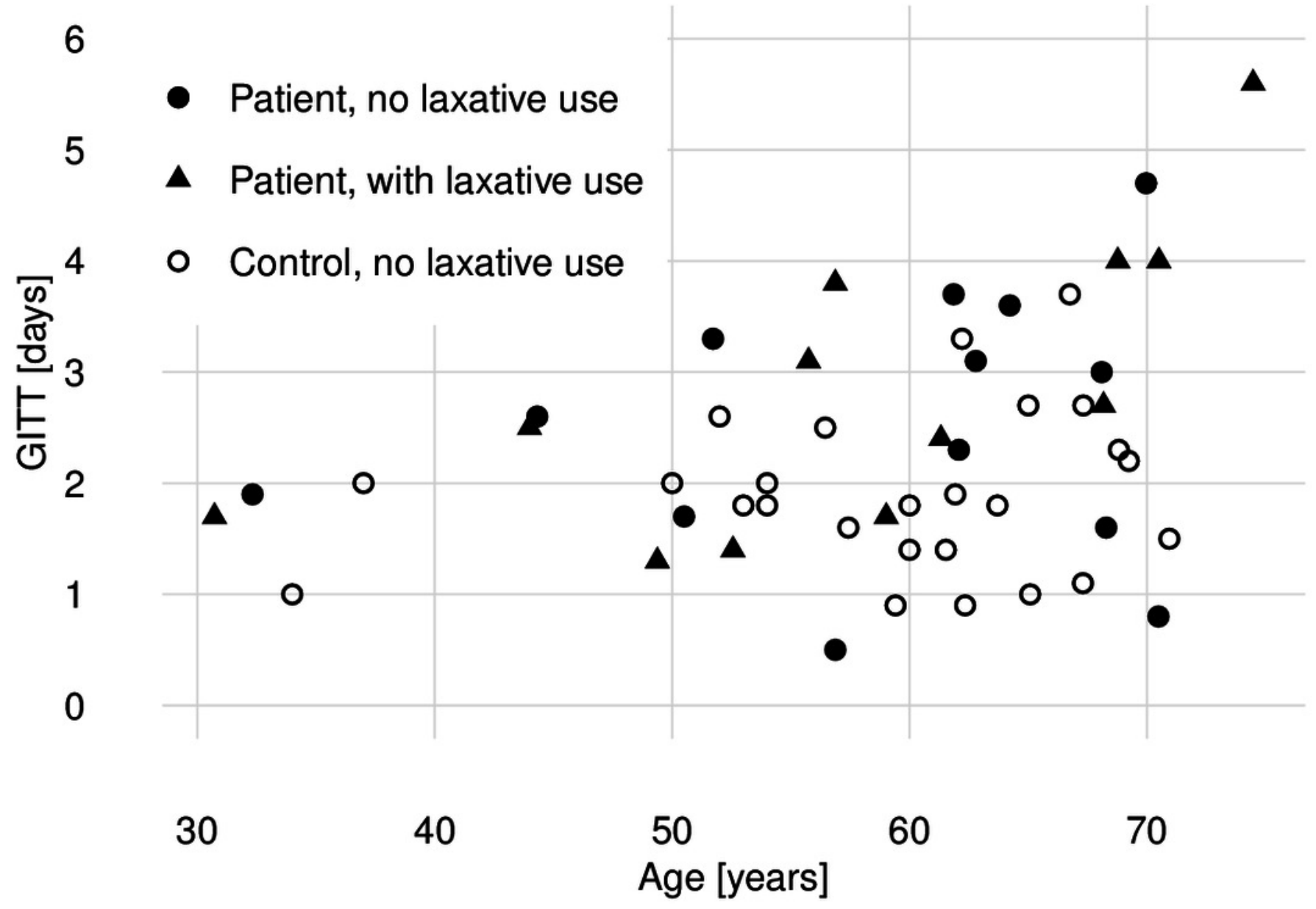




\section{Table $\mathbf{1}$ (on next page)}

Subject characteristics and results. 


\begin{tabular}{|c|c|c|c|}
\hline & $\begin{array}{l}\text { Patients with } \\
\text { acquired brain injury } \\
(n=25)\end{array}$ & Controls $(\mathrm{n}=25)$ & $\mathrm{p}$ \\
\hline Sex, males & $18(72 \%)$ & $18(72 \%)$ & 1 \\
\hline \multicolumn{4}{|l|}{ Age (Years) } \\
\hline Median (IQR) & $61.3(51.7-68.2)$ & $61.5(54.0-65.1)$ & $0.74 *$ \\
\hline \multicolumn{4}{|l|}{ Type of injury } \\
\hline Ischemic stroke & $12(48 \%)$ & - & \\
\hline Hemoragic stroke & $2(8 \%)$ & - & \\
\hline Subarachnoid hemorrhage & $7(28 \%)$ & - & \\
\hline Traumatic brain injury & $3(12 \%)$ & - & \\
\hline Anoxic brain injury & $1(4 \%)$ & - & \\
\hline \multicolumn{4}{|l|}{ Days since injury } \\
\hline Median (IQR) & $71(39-131)$ & - & \\
\hline \multicolumn{4}{|l|}{ FIM score } \\
\hline Median (IQR) & $111(97-116)$ & - & \\
\hline Laxative use & $12(48 \%)$ & $0(0 \%)$ & \\
\hline Magnesium oxide & $10(40 \%)$ & $0(0 \%)$ & \\
\hline Macrogol & $2(8 \%)$ & $0(0 \%)$ & \\
\hline Sodium picosulfate & $2(8 \%)$ & $0(0 \%)$ & \\
\hline Bisacodyl & $1(4 \%)$ & $0(0 \%)$ & \\
\hline \multicolumn{4}{|l|}{ Total GITT (days) } \\
\hline Mean (SD) & $2.68(1.24)$ & $1.92(0.73)$ & $0.011 *$ \\
\hline
\end{tabular}

1 FIM, Functional independence measure; GITT, gastrointestinal transit time.

$2 *$ Welch t-test 


\section{Table 2 (on next page)}

Multiple linear regression, modeling gastrointestinal transit time by presence of acquired brain injury ( $\mathrm{BBI} ; 25$ patients and 25 healthy controls), age and sex. 
Coefficient $(95 \% \mathrm{CI})$

$\mathrm{ABI}$

Age (years)

Male

Constant
$0.798 * *(0.249 ; 1.347)$

$0.035^{*}(0.008 ; 0.062)$

$-0.233(-0.844 ; 0.379)$

$0.025(-1.648 ; 1.698)$

\section{$1 \% \mathrm{p}<0.05 ; * * \mathrm{p}<0.01$}

$2 \quad \mathrm{R}^{2}=0.248 ;$ adjusted $\mathrm{R}^{2}=0.199$ 


\section{Table 3(on next page)}

Heart rate variability in patients with acquired brain injury.

For each patient, a 24-hour analysis, and a geometric mean of four 5 min analyses measured at $1 \mathrm{pm}, 6 \mathrm{pm}, 2$ am and 6 am was calculated. 
Geometric mean $(95 \% \mathrm{CI})$

24-hour analysis $(n=24) \quad 5$ min analysis $(n=25)$

Heart rate* $\left(\mathrm{min}^{-1}\right)$

$72.3(67.4 ; 77.1)$

$67.1(61.9 ; 72.3)$

SDNN (ms)

$126.7(106.5 ; 150.8)$

$29.3(23.7 ; 36.2)$

RMSSD (ms)

$20.5(15.2 ; 27.6)$

$18.1(13.4 ; 24.6)$

LF power $\left(\mathrm{ms}^{2}\right)$

$373.2(217.4 ; 640.6)$

$217.0(131.4 ; 358.3)$

HF power $\left(\mathrm{ms}^{2}\right)$

$126.9(72.5 ; 222.1)$

$90.7(50.5 ; 162.6)$

Total power $\left(\mathrm{ms}^{2}\right)$

13503 (8792; 20739)

$782(518 ; 1183)$

$\mathrm{LF} / \mathrm{HF}$

$2.9(2.2 ; 3.9)$

$2.4(1.7 ; 3.4)$

$1{ }^{*}$ Arithmetic mean.

2 SDNN, standard deviation of normal-to-normal intervals; RMSSD, root mean square of

3 successive differences; LF, low frequency; HF, high frequency. 
Table 4(on next page)

Correlations between the mean of four 5 min heart rate variability analyses and gastrointestinal transit time (GITT), age, and functional independence measure (FIM). 


\begin{tabular}{llll} 
& GITT (days) $)^{\dagger}$ & Age $(\text { years })^{\dagger}$ & FIM $^{*}$ \\
\hline HR & -0.027 & -0.145 & -0.388 \\
$\log ($ SDNN $)$ & -0.247 & $-0.504^{*}$ & 0.370 \\
$\log ($ RMSSD $)$ & -0.119 & -0.309 & 0.372 \\
$\log ($ HF power $)$ & -0.117 & -0.330 & 0.375 \\
$\log ($ LF power $)$ & -0.294 & $-0.528^{* *}$ & 0.350 \\
$\log ($ LF $/$ HF $)$ & -0.232 & -0.212 & -0.059 \\
\hline
\end{tabular}

$1 * \mathrm{p}<0.05 ; * * \mathrm{p}<0.01$

2 †Pearson's product-moment correlation $(r)$.

3 †Spearman’s rank correlation $(\rho)$.

4 SDNN, standard deviation of normal-to-normal intervals; RMSSD, root mean square of

5 successive differences; LF, low frequency; HF, high frequency. 